\title{
Why Not Prevent ARDS? The Possible Role of Plasma Biomarkers in Surgery
}

Cardiac, aortic vascular, and thoracic surgical patients are at high risk of developing ARDS due to direct (ie, surgical lung manipulation, high-volume positive-pressure ventilation) and indirect insults (ie, blood transfusions, systemic inflammation). Blum et al $^{1}$ identified a $0.2 \%$ incidence of new-onset ARDS in a general surgery population, whereas Kogan et $\mathrm{al}_{,}{ }^{2}$ in a retrospective analysis of 6,069 patients after cardiac surgery, found a $0.6 \%$ incidence of ARDS in cardiac surgical patients. On the microscopic level, ARDS is a result of increased capillary permeability secondary to epithelial damage from an activation of the inflammatory and coagulation cascades, which results in endothelial permeability. Not surprisingly, in previous studies in critically ill subjects with, blood and alveolar lavage showed increased levels of biomarkers for inflammation, endothelial, and epithelial injury, and showed dysregulated coagulation. With surgical patients, there is a unique opportunity to understand which biomarkers might be helpful in identifying, early on, patients who will develop ARDS and whether it is possible to prevent the devastating sequelae of ARDS.

In this issue of the Journal, Yadav et $\mathrm{al}^{3}$ performed an observational single-center case-control study of subjects who underwent elective thoracic, aortic vascular, and cardiac surgery. Subjects who developed ARDS based on the Berlin definition were matched to 2 subjects who did not develop ARDS. ${ }^{3,4}$ The aims of the study were the following: first, to evaluate whether increased levels of plasma biomarkers in the immediate postsurgical period compared with presurgery levels could predict which subjects would develop ARDS; and second, to describe what biomarkers (which pathways) developed in the early phases of ARDS. The investigators strategically selected significant time points for the plasma samples: baseline sample (before the surgery), an immediate postsurgical sample (adjusted for $2 \mathrm{~h}$ after the major insult related to the type of surgery), and the third

Dr De Santis Santiago has no conflicts to disclose. Dr Kacmarek has disclosed relationships with Medtronic, Venner Medical, and Orange Medical. Dr Berra has disclosed a relationship with Venner Medical.

DOI: $10.4187 /$ respcare.06637 sample, $4 \mathrm{~h}$ after the second sample. Among 467 enrolled subjects, 26 developed ARDS (5.6\%). The subjects were diagnosed with ARDS a median of 43.9 (interquartile range [IQR], 19.1-78.3) h after completion of the surgical procedure. The ARDS cohort had greater fluid administration and higher peak inspiratory pressure compared with a matched group of subjects without ARDS.

See the Original Study on Page 1331

To investigate which cellular type was most involved in the early development of ARDS, these authors measured plasma levels of inflammatory cytokines, endothelial, and epithelial injury biomarkers as well as coagulation biomarkers, specifically, interleukin (IL) 6 and IL-8, surfactant protein-D, von Willebrand factor, angiopoietin-2, intercellular adhesion molecule 1, plasminogen activator inhibitor-1, and soluble receptor for advanced glycation end products.

A biomarker is defined as "a characteristic that is objectively measured and evaluated as an indicator of normal biological processes, pathogenic processes or pharmacologic response to a therapeutic intervention." 5 The authors found that the subjects who developed ARDS were more likely to have early elevations in plasma inflammatory markers (eg, IL-8), epithelial injury markers (surfactant protein-D and soluble receptor for advanced glycation end products), and dysregulated coagulation marker (plasminogen activator inhibitor-1). In this study, (1) soluble receptor for advanced glycation end products was increased in absolute values $6 \mathrm{~h}$ after the surgery; a soluble receptor for advanced glycation end products, which marks alveolar epithelial injury, is associated with higher 90-d mortality in patients with ARDS6; (2) surfactant protein-D, another epithelial injury marker, and rapidly increased within $2 \mathrm{~h}$ in this study population, can help diagnosis ARDS among other medical conditions ${ }^{7}$; (3) plasminogen activator inhibitor- 1 , which was increased in absolute values at baseline, followed by an increased delta within $6 \mathrm{~h}$, is a marker for dysregulated coagulation and an acute-phase protein related to mortality ${ }^{8,9}$; (4) IL-8, a marker of systemic inflammation, was increased after surgery both 


\section{EDITORIALS}

in absolute values and when compared with the baseline. IL-8 plays a fundamental role in connecting the ARDS onset with its systemic impact by supporting the identification of ARDS subphenotypes and devoted treatments. ${ }^{10}$ However, endothelial markers were not significantly elevated, which suggests that endothelial injury is a late event in ARDS or that it plays a minor role in the development of postoperative ARDS. Early recognition of ARDS development might help guide ventilation and fluids management in these patients.

The results presented in this study are promising and, certainly, will need confirmatory reports in other and larger cohorts of surgical patients. In addition, one should realize that ARDS is a multifactorial syndrome and that postsurgical severe hypoxemia after major vascular or cardiac surgery is often the consequence of large fluid resuscitation with crystalloids and blood, atelectasis, and an intraoperative ventilatory strategy that can lead to hypoxemia and infiltrates compatible with ARDS. In fact, the authors indicated a concern with the possible misdiagnosis of ARDS, which indicated that some patients may have had cardiogenic pulmonary edema as opposed to ARDS.

This seems like a possible alternative diagnosis when considering the short duration of mechanical ventilation (ventilator-free day 26.2 [IQR, 23.2-27.7] d) and ICU length of stay (3.5 [IQR, 1.1-5.9] d) of the patients diagnosed with ARDS. Is it hard to imagine that a patient with ARDS would only require $1.1 \mathrm{~d}$ in the ICU. However, the major concern with this study is the fact that no information on patient management during the time in the ICU is provided. The authors indicated that the median time to the development of ARDS was 43.9 (IQR, 19.1-78.3) h. Clearly, in this period, ARDS may develop as a result of ventilator-induced lung injury. Unfortunately, we do not have the driving pressure (plateau pressure minus PEEP) during the insult period or while in the ICU, ${ }^{11}$ and levels of oxygenation maintained during the period of postoperative ventilator management. ${ }^{12}$ The combination of these ventilatory settings, with prediction values, and biomarkers can enrich the understanding of ARDS pathophysiology and the interaction among the possible causes.

In addition, Costa Leme et al ${ }^{13}$ showed that postoperative lung complications in cardiac surgical subjects were easily reversible once strategies such as lung recruitment maneuvers and titrated PEEP selection are applied. Thus, postsurgical ARDS diagnosis and treatment might not be generalized to other forms of ARDS. Nevertheless, Yadav et $\mathrm{al}^{3}$ shed light on how early ARDS biomarkers evaluation may help in preventing or reduc- ing ARDS onset and also guide ventilation and fluids management in this population.

\section{Roberta R De Santis Santiago MD Lorenzo Berra MD \\ Robert M Kacmarek PhD RRT FAARC \\ Harvard Medical School \\ Massachusetts General Hospital \\ Boston, Massachusetts}

\section{REFERENCES}

1. Blum JM, Stentz MJ, Dechert R, Jewell E, Engoren M, Rosenberg AL, and Park PK. Preoperative and intraoperative predictors of postoperative acute respiratory distress syndrome in a general surgical population. Anesthesiology 2013;118(1):19-29.

2. Kogan A, Preisman S, Levin S, Raanani E, Sternik L. Adult respiratory distress syndrome following cardiac surgery. J Card Surg 2014;29(1):41-46.

3. Yadav H, Bartley A, Keating S, Meade LA, Norris PJ, Carter RE, et al. Evolution of validated biomarkers and intraoperative parameters in the development of postoperative ARDS. Respir Care 2018;63(11):13311340

4. The ARDS Definition Task Force. Acute respiratory distress syndrome: the Berlin Definition. JAMA 2012;307(23):2526-2533.

5. Biomarkers Definitions Working Group. Biomarkers and surrogate endpoints: preferred definitions and conceptual framework. Clin Pharmacol Ther 2001;69(3):89-95.

6. Jabaudon M, Blondonnet R, Pereira B, Cartin-Ceba R, Lichtenstern C, Mauri T, et al. Plasma sRAGE is independently associated with increased mortality in ARDS: a meta-analysis of individual patient data. Intensive Care Med 2018;44(9):1388-1399.

7. Park J, Pabon M, Choi AMK, Siempos II, Fredenburgh LE, Baron $\mathrm{RM}$, et al. Plasma surfactant protein-D as a diagnostic biomarker for acute respiratory distress syndrome: validation in US and Korean cohorts. BMC Pulm Med 2017;17(1):204.

8. Ozolina A, Sarkele M, Sabelnikovs O, Skesters A, Jaunalksne I, Serova J, et al. Activation of coagulation and fibrinolysis in acute respiratory distress syndrome: a prospective pilot study. Front Med (Lausanne) 2016;3:64.

9. Gouda MM, Shaikh SB, Bhandary YP. Inflammatory and fibrinolytic system in acute respiratory distress syndrome. Lung 2018; 196(5):609-616.

10. Calfee CS, Delucchi K, Parsons PE, Thompson BT, Ware LB, Matthay MA, NHLBI ARDS Network. Subphenotypes in acute respiratory distress syndrome: latent class analysis of data from two randomised controlled trials. Lancet Respir Med 2014;2(8):611-620.

11. Neto AS, Hemmes SN, Barbas CS, Beiderlinden M, FernandezBustamante A, Futier E, et al; PROVE Network Investigators. Association between driving pressure and development of postoperative pulmonary complications in patients undergoing mechanical ventilation for general anaesthesia: a meta-analysis of individual patient data. Lancet Respir Med 2016;4(4):272-280.

12. Esteve F, Lopez-Delgado JC, Javierre C, Skaltsa K, Carrio ML, Rodríguez-Castro D, et al. Evaluation of the $\mathrm{PaO} 2 / \mathrm{FiO} 2$ ratio after cardiac surgery as a predictor of outcome during hospital stay. BMC Anesthesiol 2014;14:83.

13. Costa Leme A, Hajjar LA, Volpe MS, Fukushima JT, De Santis Santiago RR, Osawa EA, et al. Effect of intensive vs moderate alveolar recruitment strategies added to lung-protective ventilation on postoperative pulmonary complications: a randomized clinical trial. JAMA 2017;317(14):1422-1432. 